\title{
Electron plasmas as a diagnostic tool for hyperfine spectroscopy of antihydrogen
}

T. Friesen ${ }^{\mathrm{a}}$, C. Amole ${ }^{\mathrm{b}}$, M. D. Ashkezaric ${ }^{\mathrm{c}}$ M. Baquero-Ruiz ${ }^{\mathrm{d}}$, W. Bertsche $^{\mathrm{e}, \mathrm{f}}$, P. D. Bowe ${ }^{\mathrm{g}}$, E. Butler ${ }^{\mathrm{h}}$, A. Capra ${ }^{\mathrm{b}}$, C. L. Cesar ${ }^{\mathrm{i}}$, M. Charlton ${ }^{\mathrm{j}}$, A. Deller, S. Eriksson ${ }^{\mathrm{j}}$, J. Fajans ${ }^{\mathrm{d}}$, M. C. Fujiwarak, ${ }^{\mathrm{k}, \mathrm{a}}$, D. R. Gill ${ }^{\mathrm{k}}$, A. Gutierrez $^{1}$, J. S. Hangst ${ }^{\mathrm{g}}$, W. N. Hardy ${ }^{1}$, M. E. Hayden ${ }^{\mathrm{c}}$, C. A. Isaac ${ }^{\mathrm{j}}, \mathrm{S}$. Jonsell $^{\mathrm{m}}$, L. Kurchaninov ${ }^{\mathrm{k}}$, A. Little ${ }^{\mathrm{d}}$, N. Madsen ${ }^{\mathrm{j}}$, J. T. K. McKenna ${ }^{\mathrm{n}}$, S. Menary $^{\mathrm{b}}$, S. C. Napoli ${ }^{\mathrm{j}}$, K. Olchanski ${ }^{\mathrm{k}}$, A. Olin ${ }^{\mathrm{k}}$, P. Pusa ${ }^{\mathrm{n}}$, C. Ø. Rasmussen $^{\mathrm{g}}$, F. Robicheaux ${ }^{\mathrm{o}}$, E. Sarid ${ }^{\mathrm{p}}$, D. M. Silveira ${ }^{\mathrm{i}}$, C. So ${ }^{\mathrm{d}}, \mathrm{S}$. Stracka $^{\mathrm{k}}$, R. I. Thompson ${ }^{\mathrm{a}}$, D. P. van der Werf ${ }^{\mathrm{j}}$ and J. S. Wurtele ${ }^{\mathrm{d}}$

${ }^{a}$ Department of Physics and Astronomy, University of Calgary, Calgary AB, T2N 1N4, Canada

${ }^{b}$ Department of Physics and Astronomy, York University, Toronto ON, M3J 1P3, Canada

${ }^{c}$ Department of Physics, Simon Fraser University, Burnaby BC, V5A 1S6, Canada

${ }^{d}$ Department of Physics, University of California, Berkeley, CA 94720-7300, USA

e School of Physics and Astronomy, University of Manchester, M13 9PL Manchester, UK

${ }^{f}$ The Cockcroft Institute, WA4 4AD Warrington, UK

${ }^{g}$ Department of Physics and Astronomy, Aarhus University, DK-8000 Aarhus C, Denmark

${ }^{h}$ Physics Department, CERN, CH-1211 Geneva 23, Switzerland

${ }^{i}$ Instituto de Física, Universidade Federal do Rio de Janeiro, Rio de Janeiro 21941-972, Brazil

${ }^{j}$ Department of Physics, Swansea University, Swansea SA2 8PP, UK

${ }^{k}$ TRIUMF, 4004 Wesbrook Mall, Vancouver BC, V6T 2A3, Canada

${ }^{l}$ Department of Physics and Astronomy, University of British Columbia, Vancouver BC, V6T 1Z4, Canada

${ }^{m}$ Department of Physics, Stockholm University, SE-10691 Stockholm, Sweden

${ }^{n}$ Department of Physics, University of Liverpool, Liverpool L69 7ZE, UK

${ }^{\circ}$ Department of Physics, Auburn University, Auburn, AL 36849-5311, USA

${ }^{p}$ Department of Physics, NRCN-Nuclear Research Center Negev, Beer Sheva, 84190, Israel

\begin{abstract}
.
Long term magnetic confinement of antihydrogen atoms has recently been demonstrated by the ALPHA experiment at CERN, opening the door to a range of experimental possibilities. Of particular interest is a measurement of the antihydrogen spectrum. A precise comparison of the spectrum of antihydrogen with that of hydrogen would be an excellent test of CPT symmetry. One prime candidate for precision CPT tests is the ground-state hyperfine transition; measured in hydrogen to a precision of nearly one part in $10^{12}$. Effective execution of such an experiment with trapped antihydrogen requires precise knowledge of the magnetic environment.

Here we present a solution that uses an electron plasma confind in the antihydrogen trapping region. The cyclotron resonance of the electron plasma is probed with microwaves at the cyclotron frequency and the subsequent heating of the electron plasma is measured through the plasma quadrupole mode frequency. Using this method, the minimum magnetic field of the neutral trap can be determined to within 4 parts in $10^{4}$. This technique was used extensively in the recent demonstration of resonant interaction with the hyperfine levels of trapped antihydrogen atoms.
\end{abstract}

Keywords: Antihydrogen, Non-neutral Plasmas, Penning Traps

PACS: $32.30 .-r, 52.27 . J t, 52.50 . \mathrm{Sw}$ 


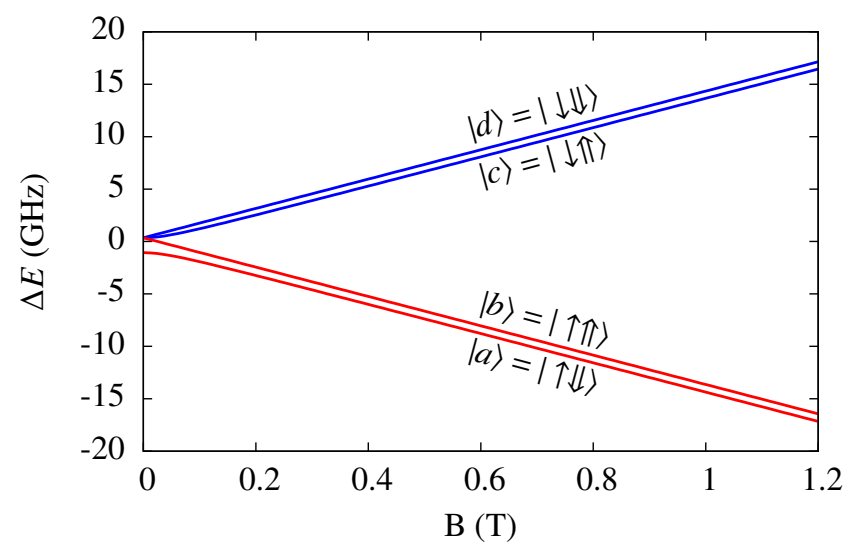

FIGURE 1. Breit-Rabi diagram for the ground state of hydrogen (and antihydrogen if CPT invariance holds) showing the relative energy levels, in frequency units, in a magnetic field. The arrows in the state vectors denote the positron (single arrow) and antiproton (double arrow) spins in the high field limit.

\section{INTRODUCTION}

The hydrogen atom is one of the best studied and understood systems in physics. Precision measurements of its spectra have a long and distinguished history and continue to improve upon the accuracy of fundamental constants [1]. It is therefore natural that study of its antimatter counterpart, antihydrogen, is of fundamental interest. In particular, because of the precision achieved with the hydrogen spectrum, measurements of the antihydrogen spectrum have the potential to be excellent tests of CPT (charge conjugation, parity inversion, and time reversal) symmetry [2]. The ground state hyperfine transition, giving rise to the famous $21-\mathrm{cm}$ line in radio-astronomy, is one of the most promising candidates for such a test as it has been measured with a precision of 1.4 parts in $10^{12}$ in hydrogen [3].

Recently, the ALPHA collaboration reported the first resonant quantum transitions between the hyperfine levels of antihydrogen [4]. Unlike the seminal experiments on hydrogen, where a hydrogen maser was used, we must employ very different experimental techniques that avoid annihilation of antihydrogen from wall collisions. The ALPHA experiment uses a magnetic minimum neutral atom trap to confine the antihydrogen via its magnetic moment, $\mu_{0}$. The trapped antiatoms can be stored and interacted with for long periods of time (currently up to $1000 \mathrm{~s} \mathrm{[5]).}$

In a strong magnetic field the ground state is split into two pairs of states (see Fig. 1). States $|d\rangle$, and $|c\rangle$ are 'low-field seeking states' and states $|a\rangle$ and $|b\rangle$ are high field seeking states and unconfined by the neutral atom trap. Once trapped, microwave radiation can be injected into the trap to induce transitions from $|d\rangle \rightarrow|a\rangle$ and $|c\rangle \rightarrow|b\rangle$, which in the high magnetic field correspond to positron spin flips. The untrapped atoms will escape and annihilate on the surrounding apparatus, which can be detected by a three-layer imaging silicon detector [6].

The neutral atom trap fields, which scale as $z^{2}$ axially and $r^{3}$ radially, are most homogeneous at the trap minimum. Microwaves tuned precisely to the minimum resonance frequency therefore have the highest probability of inducing a transition since an an- 
tihydrogen atom will remain in resonance for the maximum length of time. To hit the minimum resonance frequency one requires an accurate measurement of the minimum magnetic field.

\section{TECHNIQUE}

We measure the magnetic field using the cyclotron resonance of an electron plasma stored in the antihydrogen trapping region. Microwaves are injected near the electron cyclotron frequency, heating the plasma. The electron cyclotron resonance lineshape can be mapped out by measuring the plasma heating as a function of microwave frequency. In the case of a uniform magnetic field across the plasma, heating of the electron plasma will be maximized when the microwave frequency matches the cyclotron resonance. In ALPHA, plasma temperatures are typically measured by slowly lowering the confining well of the plasma and releasing the particles onto a micro-channel plate and phosphor screen detector. One can measure the energy distribution in this manner and extract the plasma temperature [7]. The downside to this technique is that the plasma is lost in the process. This is problematic for measuring cyclotron resonance because: (1) Heating at each microwave frequency is measured on a different electron plasma, adding uncertainty and, (2) preparing and dumping an electron plasma for each frequency point is time consuming. For these reasons, as well as hardware limitations, we instead implemented a non-destructive temperature measurement based on the quadrupole mode of the electron plasma.

\section{Quadrupole Mode}

Non-neutral plasmas in a Penning trap form spheroids that undergo normal modes of oscillation at frequencies that depend on the plasma properties. In the cold-fluid limit the frequency of these modes can be solved analytically [8]. Experimental studies of these modes in ion, electron and positron plasmas have shown good agreement with the theory $[9,10,11]$ and have been used as diagnostics of density and aspect ratio [12]. The second order, azimuthally symmetric, mode is known as the quadrupole mode and is of particular interest because the mode frequency depends on the plasma temperature. The quadrupole mode, which is an oscillation of the plasma aspect ratio, can therefore be used as measure of the temperature change of an electron plasma heated by cyclotron radiation. An approximate temperature correction to the cold-fluid frequency exists and has been shown to agree well with experiment $[13,12]$. Using this approximate form, a temperature change $\Delta T$ will result in a quadrupole mode frequency change given by:

$$
\left(\omega_{2}^{\prime}\right)^{2}-\left(\omega_{2}\right)^{2}=20\left(3-\frac{\alpha^{2}}{2} \frac{\omega_{p}^{2}}{\left(\omega_{2}^{c}\right)^{2}} \frac{\partial^{2} f(\alpha)}{\partial \alpha^{2}}\right) \frac{k_{B} \Delta T}{m L^{2}},
$$

where $\omega_{p}=\sqrt{\frac{n e^{2}}{m \epsilon_{0}}}$ is the plasma frequency, $\omega_{2}$ and $\omega_{2}^{\prime}$ are the quadrupole mode frequencies before and after heating, respectively, and $\omega_{2}^{c}$ is the cold-fluid result. Equation (1) 


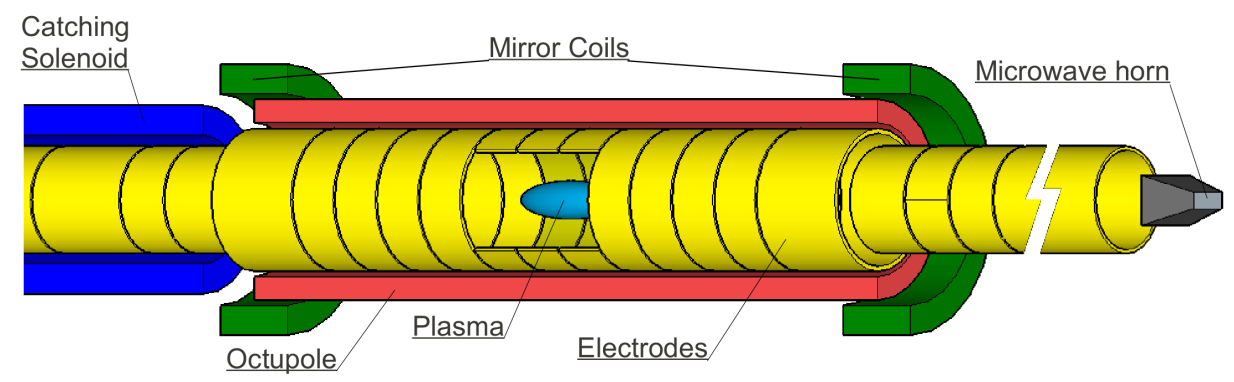

FIGURE 2. Sketch of the mixing region of the ALPHA apparatus. Not shown is a $1 \mathrm{~T}$ superconducting solenoid surrounding this entire region. The blue spheroid in the centre of the trap indicates the plasma position. The radial extent of the plasma is much smaller in practice than shown.

also contains a geometry dependent term given by $f(\alpha)=2 Q_{1}\left[\alpha\left(\alpha^{2}-1\right)^{-1 / 2}\right] /\left(\alpha^{2}-1\right)$. For the typical plasmas used in the current work $\Delta \omega_{2} \ll \omega_{2}$ and Eqn. (1) can be approximated as $\Delta \omega_{2}=\beta \Delta T$, where $\beta$ is a function of plasma density, radius, and length.

The quadrupole mode frequency can be measured non-destructively by applying a drive pulse to an electrode near the plasma at the quadrupole frequency. Immediately after the drive pulse is turned off, the ringing of the plasma can be picked up and the ringing frequency measured (see apparatus section for more details). By doing this periodically we can monitor the quadrupole mode frequency over time and measure the frequency jump due to a pulse of microwaves near the cyclotron resonance. Because this is a non-destructive measurement, the cyclotron resonance lineshape can be mapped out using a single plasma in a relatively short time.

\section{APPARATUS}

The core of the ALPHA apparatus is a cylindrical Penning trap for charged particle confinement, surrounded by superconducting magnets in an variation of the Ioffe-Pritchard [14] configuration that form a magnetic minimum trap for neutral antihydrogen (see Fig. 2 ). The Penning trap consists of cylindrical electrodes that confine charged particles axially with the application of voltages and a uniform solenoidal field directed along the electrode axis that keeps the particles radially confined. The Penning trap is used to capture, store, manipulate, and mix antiprotons and positrons to form antihydrogen. Mixing occurs at the minimum of the neutral atom trap magnetic field produced by two mirror coils, creating an axially increasing field, and a transverse octupole (the traditional magnetic trap uses a quadrupole) that creates a radially increasing field [15, 16]. Antihydrogen that is formed with a kinetic energy of less than $0.5 \mathrm{~K}$ in temperature units will be trapped.

The properties of the electron, antiproton and positron plasmas are important factors in the successful production of trappable antihydrogen. In ALPHA, particle number is measured by releasing the plasmas onto a Faraday cup at one end of the trap and measuring the charged deposited. The integrated radial profile of the plasmas can be measured by dumping the plasma onto a micro-channel plate and phosphor screen 


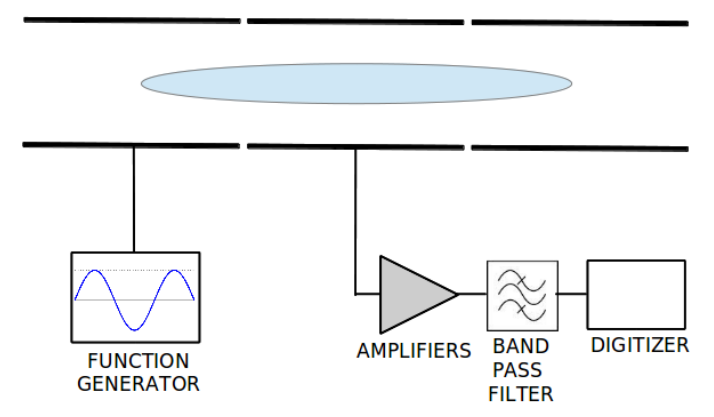

FIGURE 3. Sketch of the quadrupole mode detection circuit.

detector [17] (for convenience the whole assembly is referred to as 'MCP detector' from here on). From the particle number, radial profile and knowledge of the confining potentials we can determine the plasma density and length. Plasma temperature can also be measured using the MCP detector by slowly lowering the confining potential and measuring the energy distribution of the particles that are released [7].

The quadrupole mode frequency is measured by first applying a Gaussian modulated sinusoidal pulse near the quadrupole mode frequency to an electrode at one end of the plasma (see Fig. 3). Following the drive pulse, the resulting plasma ringing at the quadrupole mode frequency is picked up on an electrode centred on the plasma, amplified, and filtered before being digitized. A Fast-Fourier-Transform is applied to the digitized signal and a peak finding routine finds the quadrupole mode frequency. Using this system the quadrupole mode frequency is measured at a rate of roughly $1 \mathrm{~s}^{-1}$.

\section{RESULTS}

\section{Quadrupole mode}

The linearity of the quadrupole mode shift against the temperature change of the plasma must be experimentally confirmed since any non-linearity would distort the measured lineshapes. We can effectively calibrate the quadrupole frequency shift against the plasma temperature change by destructively measuring the plasma temperature with the MCP detector. The typical electron plasmas used here consists of $8 \times 10^{6}$ to $7 \times 10^{7}$ electrons in a roughly harmonic well spanning three electrodes (Fig. 3). The plasmas have lengths from $20-40 \mathrm{~mm}$, radii of $1-2 \mathrm{~mm}$, and quadrupole mode frequencies around $26 \mathrm{MHz}$.

To calibrate the quadrupole frequency shift, the plasma is heated by applying a 'white' noise drive to a nearby electrode, while constantly monitoring the quadrupole mode frequency. After the plasma has reached a new thermal equilibrium we destructively measure the plasma temperature. Figure 4 plots the resulting final plasma temperatures vs the quadrupole mode shifts for plasmas of different aspect ratios. The initial plasma temperature from load to load is consistent to within $20 \mathrm{~K}$. The measured calibrations confirm that the quadrupole frequency shifts are linear with the temperature change of 


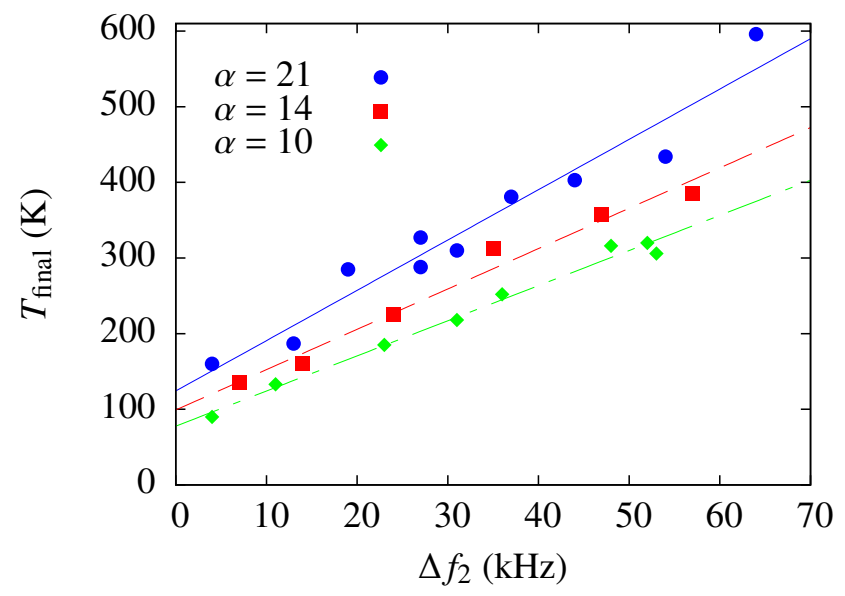

FIGURE 4. Final plasma temperature versus the corresponding quadrupole mode frequency shift for plasmas with three different aspect ratios.

the plasma.

\section{Electron cyclotron measurements}

We measure the cyclotron resonance of an electron plasma by loading the plasma into the centre of the antihydrogen trapping region and continuously measuring the quadrupole mode frequency. We apply a series of microwave pulses at frequencies scanning across the cyclotron resonance and the resulting quadrupole frequency jump is measured for each frequency. Each microwave pulse is typically $4 \mu \mathrm{s}$ in length and a delay of 15 - $30 \mathrm{~s}$ between pulses is imposed so that the plasma cools back to its equilibrium temperature between each pulse. By ensuring the plasma is at the same temperature when each pulse is injected, we avoid a variable temperature broadening distorting the lineshape.

\section{Uniform field}

We first measure the electron cyclotron resonance without the neutral atom trap engaged. In this case the magnetic field is simply the uniform magnetic field due to the Penning trap solenoid. Figure 5(a) shows an example of the real-time quadrupole frequency read-out during a cyclotron scan. We take the magnitude of the quadrupole frequency jump as a function of microwave frequency to plot a lineshape (Fig. 5(b)). The constant decrease of the quadrupole mode frequency over time is consistent the plasma expanding very slightly during the lineshape measurement. The width of the lineshape is expected to be due to thermal broadening but because we do not know the mode structure of the microwave field in the Penning trap we cannot directly extract a temperature from the width. We can confirm that the central resonance behaves as expected by changing 

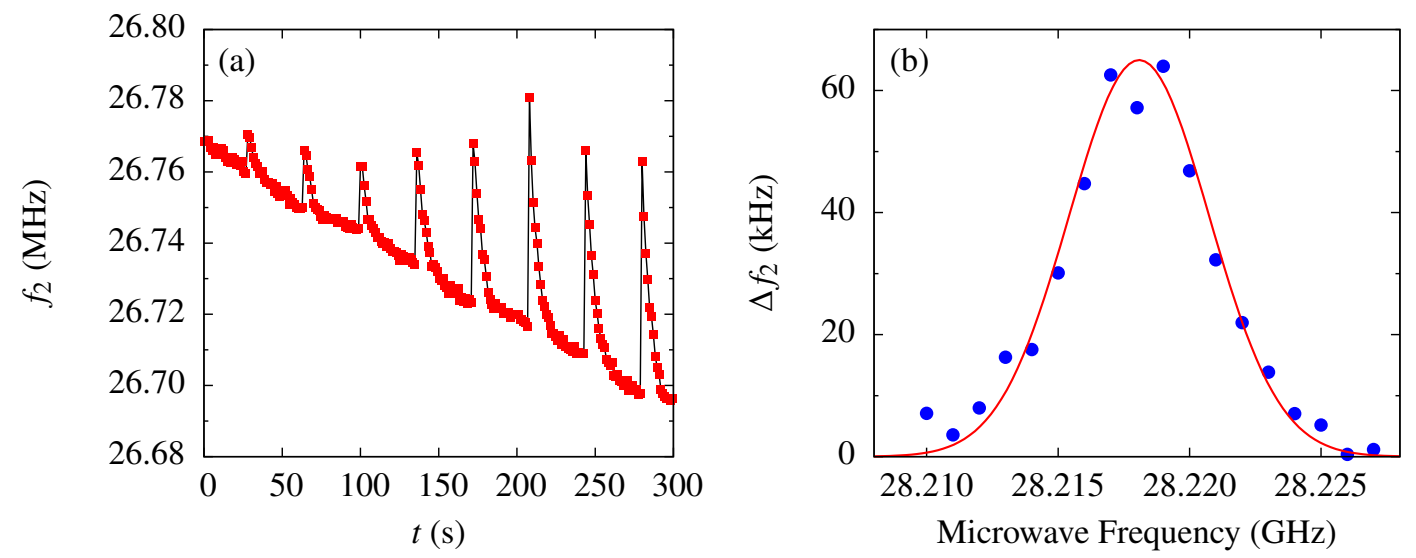

FIGURE 5. Example of the real-time quadrupole mode frequency readout (a) during a cyclotron resonance scan in a uniform magnetic field. The frequency jumps are extracted and plotted against the microwave frequency to construct the cyclotron lineshape (b).

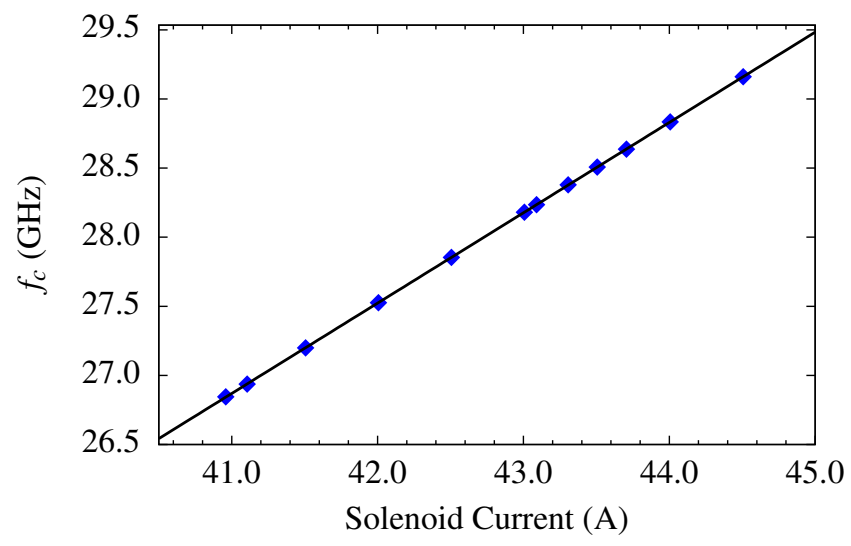

FIGURE 6. Scaling of peak frequency vs solenoid current (without neutral atom trap fields).

the Penning trap solenoid current and measuring the resonance at each field. Figure 6 shows that the measured resonance tracks the magnet current extremely well.

\section{Neutral atom trap field}

Our primary goal is to measure the minimum of the magnetic field with the neutral atom trap energized. The magnetic field is most uniform at the minimum of the neutral atom trap. By tuning the microwave frequency to the hyperfine transition frequencies at the trap minimum we maximize the transition probability. We measure the minimum magnetic field by loading an electron plasma into the centre of the antihydrogen trapping region, energizing the trapping magnets, and measuring the lineshape as described above. Near the radial centre of the trap, the field from the mirror coils will dominate the 


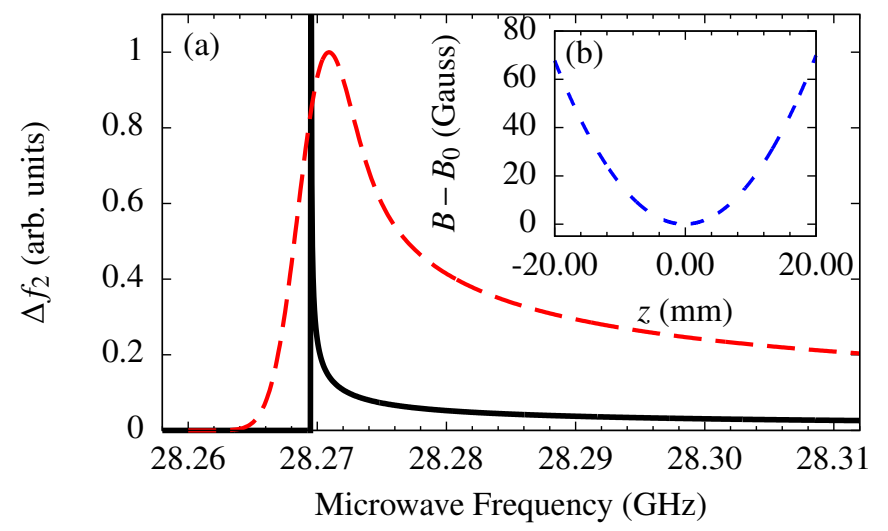

FIGURE 7. (a) Plot of the expected cyclotron resonance lineshape of an electron plasma in the mirror field without (solid black) and with (dashed red) temperature broadening. (b) The inset shows the magnetic field as a function of z-position.

field profile. The expected cyclotron lineshape in the mirror coil magnetic field is plotted in Fig. 7. The strong onset peak (solid back) marks the minimum cyclotron resonance where the largest portion of the plasma is in resonance. If we include the effect of thermal broadening the base lineshape is convolved with a Gaussian, broadening the onset peak (dashed red). As the plasma temperature increases, the peak heating frequency is also shifted further above the true minimum cyclotron resonance.

Unfortunately the situation is complicated by the microwave field in the Penning trap. The electrode structure, which includes two changes in radius (see Fig. 2) and breaks between each electrode, can give rise to complicated standing and travelling waves. The result is that the electric field strength can vary dramatically as a function of frequency and position within the trap. This distorts the measured lineshape and can result in shifts of the onset peak frequency. Figure 8 shows an example of the lineshapes measured in the mirror coil field. The strong onset peak is present but following this peak the lineshape is dominated by the spatial and frequency variations of the microwave electric field. Fortunately, the onset peak remains a strong feature and the peak frequency can still be used to measure the minimum of the magnetic trap field. Because of the uncertainty due to the microwave electric field we take the onset peak frequency as a measurement of the minimum cyclotron resonance. The systematic shift due to thermal broadening is dominated by the microwave field uncertainty and isn't account for here. Figure 9 plots the onset peak frequency as a function of the current in the mirror coils (the coils can be controlled individually but we generally use them together). As the currents are increased, both the minimum of the magnetic field and the magnetic gradient across the plasma are increased. The changing microwave field will distort the measured lineshape, shifting the onset peak frequency, in an unknown way at each current. Despite these effects we find that the onset peak frequency tracks the magnet current extremely well. We estimate the uncertainty of the minimum magnetic field measurement to be approximately $10 \mathrm{MHz}$ in frequency or 3.6 Gauss in magnetic field (3.6 parts in $10^{4}$ ). 


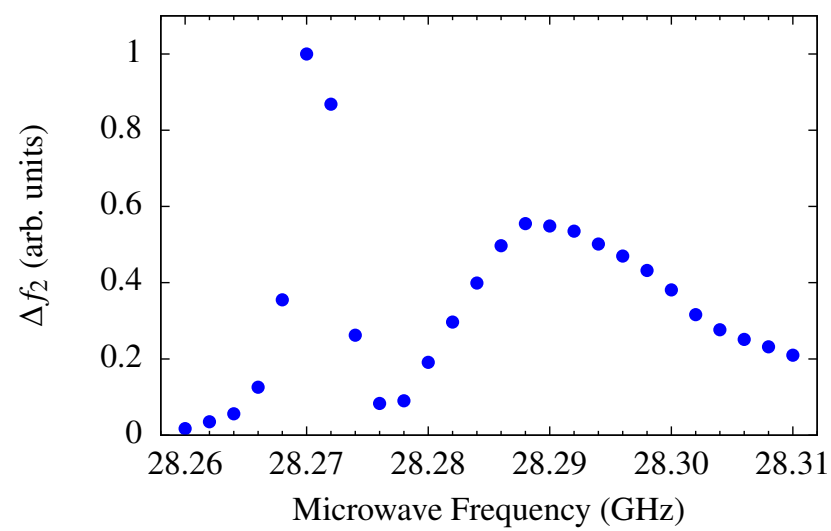

FIGURE 8. Measured cyclotron resonance lineshape in the mirror coil field.

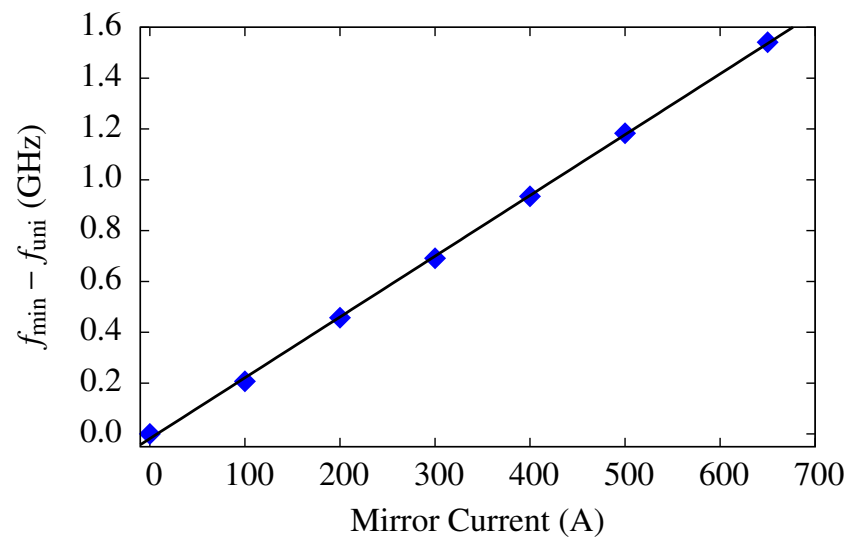

FIGURE 9. Frequency of the onset peak as a function of current in the two mirror coils. The measured resonance in the uniform field has been subtracted from the y-axis.

\section{FUTURE WORK}

These techniques were extensively used in the recent demonstration of resonant interaction with the hyperfine levels of antihydrogen. In that experiment, a binary on/off resonance measurement was made with frequency sweeps of $15 \mathrm{MHz}$ to increase the probability of inducing transitions. The next step is to measure the resonance lineshapes of the $|c\rangle \rightarrow|b\rangle$ and $|d\rangle \rightarrow|a\rangle$ transitions to more accurately determine the zero-field hyperfine splitting. In addition we will measure the antiproton spin flip transition, $|d\rangle \rightarrow|c\rangle$. This transition has the advantage of passing through a turning point in transition frequency at $0.65 \mathrm{~T}$, making it much less sensitive to magnetic field variations.

These measurements will be carried out on a new apparatus currently being built and commissioned at CERN. One of the key features of the new apparatus is the move from a single Penning trap to a two trap setup; one an antiproton 'catching' trap and the other for antihydrogen synthesis, trapping and spectroscopy (know as the 'atom' trap). The atom trap will include laser access for $1 \mathrm{~S}-2 \mathrm{~S}$ spectroscopy and potential laser cooling 
of antihydrogen. The atom trap will also feature three additional mirror coils, which can be used to flatten the axial trapping field. A flatter magnetic field will increase the precision to which we can measure antihydrogen's hyperfine spectrum. Plans are also being made to include microwave resonators in the Penning trap. A resonator is required to excite the antiproton spin flip transitions since those wavelengths $(\lambda \approx 46 \mathrm{~cm})$ will not propagate in the Penning trap electrodes. An additional resonator is being considered for frequencies in the range of the positron spin flip transitions as discussed here. This resonator would allow us to work with a known microwave mode structure and enhance the positron spin flip transition rates.

The biggest source of uncertainty in the cyclotron resonance measurements presented here is the unknown, and highly variable, nature of the microwave electric field. Some uncertainty may be removed by flattening the axial trapping magnetic field using the additional three mirror coils planned in the new apparatus. By flattening the magnetic field we increase the portion of the plasma in resonance at the minimum and approach the uniform field case. A more complete solution to this problem is the inclusion of a microwave resonator. With a microwave resonator at the appropriate frequencies, we would be working with a known electric field strength over the length of the plasma, eliminating the dominant source of uncertainty. In addition, if the entire plasma sits between two nodes of the cavity standing wave pattern, the electrons will not experience a Doppler shift of the injected microwaves. By eliminating the microwave field uncertainty and the thermal broadening, the magnetic field probe presented here can achieve a much higher sensitivity.

\section{SUMMARY}

We have implemented an in-situ method for measuring the magnetic field seen in a Penning trap. In particular we demonstrated that the minimum field in out magnetic neutral atom trap can be measured to within 4 parts in $10^{4}$. Measurement of this field is key for performing hyperfine spectroscopy on the ground state of trapped antihydrogen. The magnetic environment is measured using an electron plasma stored in the antihydrogen trapping region. The quadrupole mode of the electron plasma is used to nondestructively map out the cyclotron resonance and extract the magnetic field strength. This method was used extensively in the recent demonstration of induced transitions between ground state hyperfine levels of trapped antihydrogen. Hardware improvements in the next generation of the ALPHA experiment will allow for much higher resolution measurements of both magnetic field and the antihydrogen hyperfine spectrum.

\section{REFERENCES}

1. T. W. Hänsch, Rev. Mod. Phys. 78, 1297-1309 (2006).

2. M. H. Holzscheiter, M. Charlton, and M. M. Nieto, Physics Reports 402, 1 - 101 (2004), ISSN 0370-1573.

3. H. Hellwig, R. F. C. Vessot, M. W. Levine, P. W. Zitzewitz, D. W. Allan, and D. J. Glaze, Instrumentation and Measurement, IEEE Transactions on 19, 200 -209 (1970), ISSN 0018-9456. 
4. C. Amole, M. D. Ashkezari, M. Baquero-Ruiz, W. Bertsche, P. D. Bowe, E. Butler, A. Capra, C. L. Cesar, M. Charlton, A. Deller, P. H. Donnan, S. Eriksson, J. Fajans, T. Friesen, M. C. Fujiwara, D. R. Gill, A. Gutierrez, J. S. Hangst, W. N. Hardy, M. E. Hayden, A. J. Humphries, C. A. Isaac, S. Jonsell, L. Kurchaninov, A. Little, N. Madsen, J. T. K. McKenna, S. Menary, S. C. Napoli, P. Nolan, K. Olchanski, A. Olin, P. Pusa, C. O. Rasmussen, F. Robicheaux, E. Sarid, C. R. Shields, D. M. Silveira, S. Stracka, C. So, R. I. Thompson, D. P. van der Werf, and J. S. Wurtele, Nature 483, 439-443 (2012), ISSN 0028-0836.

5. G. B. Andresen, M. D. Ashkezari, M. Baquero-Ruiz, W. Bertsche, P. D. Bowe, E. Butler, C. L. Cesar, M. Charlton, A. Deller, S. Eriksson, J. Fajans, T. Friesen, M. C. Fujiwara, D. R. Gill, A. Gutierrez, J. S. Hangst, W. N. Hardy, R. S. Hayano, M. E. Hayden, A. J. Humphries, R. Hydomako, S. Jonsell, S. L. Kemp, L. Kurchaninov, N. Madsen, S. Menary, P. Nolan, K. Olchanski, A. Olin, P. Pusa, C. Ø. Rasmussen, F. Robicheaux, E. Sarid, D. M. Silveira, C. So, J. W. Storey, R. I. Thompson, D. P. van der Werf, J. S. Wurtele, and Y. Yamazaki, Nature Physics 7, 558-564 (2011), 1104 . 4982.

6. G. Andresen, M. Ashkezari, W. Bertsche, P. Bowe, E. Butler, C. Cesar, S. Chapman, M. Charlton, A. Deller, S. Eriksson, J. Fajans, T. Friesen, M. Fujiwara, D. Gill, A. Gutierrez, J. Hangst, W. Hardy, M. Hayden, R. Hayano, A. Humphries, R. Hydomako, S. Jonsell, L. Jørgensen, L. Kurchaninov, N. Madsen, S. Menary, P. Nolan, K. Olchanski, A. Olin, A. Povilus, P. Pusa, E. Sarid, S. S. el Nasr, D. Silveira, C. So, J. Storey, R. Thompson, D. van der Werf, and Y. Yamazaki, Nuclear Instruments and Methods in Physics Research Section A: Accelerators, Spectrometers, Detectors and Associated Equipment 684, 73 - 81 (2012), ISSN 0168-9002.

7. D. L. Eggleston, C. F. Driscoll, B. R. Beck, A. W. Hyatt, and J. H. Malmberg, Physics of Fluids B: Plasma Physics 4, 3432-3439 (1992).

8. D. H. E. Dubin, Phys. Rev. Lett. 66, 2076-2079 (1991).

9. D. J. Heinzen, J. J. Bollinger, F. L. Moore, W. M. Itano, and D. J. Wineland, Phys. Rev. Lett. 66 , 2080-2083 (1991).

10. J. J. Bollinger, D. J. Heinzen, F. L. Moore, W. M. Itano, D. J. Wineland, and D. H. E. Dubin, Phys. Rev. A 48, 525-545 (1993).

11. C. S. Weimer, J. J. Bollinger, F. L. Moore, and D. J. Wineland, Phys. Rev. A 49, 3842-3853 (1994).

12. M. Amoretti, C. Amsler, G. Bonomi, A. Bouchta, P. D. Bowe, C. Carraro, C. L. Cesar, M. Charlton, M. Doser, V. Filippini, A. Fontana, M. C. Fujiwara, R. Funakoshi, P. Genova, J. S. Hangst, R. S. Hayano, L. V. Jørgensen, V. Lagomarsino, R. Landua, D. Lindelöf, E. L. Rizzini, M. Macrí, N. Madsen, G. Manuzio, P. Montagna, H. Pruys, C. Regenfus, A. Rotondi, G. Testera, A. Variola, and D. P. van der Werf, Phys. Rev. Lett. 91, 055001 (2003).

13. M. D. Tinkle, R. G. Greaves, and C. M. Surko, Physics of Plasmas 2, 2880-2894 (1995).

14. D. E. Pritchard, Phys. Rev. Lett. 51, 1336-1339 (1983).

15. J. Fajans, W. Bertsche, K. Burke, S. F. Chapman, and D. P. van der Werf, Phys. Rev. Lett. 95, 155001 (2005).

16. G. Andresen, W. Bertsche, A. Boston, P. D. Bowe, C. L. Cesar, S. Chapman, M. Charlton, M. Chartier, A. Deutsch, J. Fajans, M. C. Fujiwara, R. Funakoshi, D. R. Gill, K. Gomberoff, J. S. Hangst, R. S. Hayano, R. Hydomako, M. J. Jenkins, L. V. Jørgensen, L. Kurchaninov, N. Madsen, P. Nolan, K. Olchanski, A. Olin, A. Povilus, F. Robicheaux, E. Sarid, D. M. Silveira, J. W. Storey, H. H. Telle, R. I. Thompson, D. P. van der Werf, J. S. Wurtele, and Y. Yamazaki, Phys. Rev. Lett. 98, 023402 (2007).

17. G. B. Andresen, W. Bertsche, P. D. Bowe, C. C. Bray, E. Butler, C. L. Cesar, S. Chapman, M. Charlton, J. Fajans, M. C. Fujiwara, D. R. Gill, J. S. Hangst, W. N. Hardy, R. S. Hayano, M. E. Hayden, A. J. Humphries, R. Hydomako, L. V. Jørgensen, S. J. Kerrigan, L. Kurchaninov, R. Lambo, N. Madsen, P. Nolan, K. Olchanski, A. Olin, A. P. Povilus, P. Pusa, E. Sarid, S. S. E. Nasr, D. M. Silveira, J. W. Storey, R. I. Thompson, D. P. van der Werf, and Y. Yamazaki, Review of Scientific Instruments 80, 123701 (2009). 